\title{
Correlations between Total Antioxidant Capacity and 8-Hydroxydeoxyguanosine with Carotid-Femoral Pulse Wave Velocity in Chronic Kidney Disease
}

\author{
Mochamad Yusuf Alsagaff ${ }^{1, *}$, Budi Susetyo Pikir ${ }^{1}$, Mochammad Thaha ${ }^{2}$, Hendri Susilo ${ }^{1}$ \\ ${ }^{1}$ Department of Cardiology and Vascular Medicine, Faculty of Medicine, Universitas Airlangga, Jl. Mayjen Prof. Dr. Moestopo No.47, \\ Surabaya 60132, Indonesia \\ ${ }^{2}$ Department of Internal Medicine, Faculty of Medicine, Universitas Airlangga, Jl. Mayjen Prof. Dr. Moestopo No.47, Surabaya 60132, Indonesia \\ *Corresponding author. E-mail: yusuf_505@fk.unair.ac.id
}

Received date: Apr 20, 2020; Revised date: Jul 17, 2020; Accepted date: Aug 4, 2020

\section{Abstract}

2 ACKGROUND: Chronic kidney disease (CKD) is often associated with an increased risk of cardiovascular disease. Cardiovascular disease in CKD can be affected by oxidative stress and inflammation. The oxidative stress can affect arterial stiffness, especially in patients with CKD, which will increase the risk of cardiovascular disease. This study aimed to explain the correlation between total antioxidant capacity (TAC) and 8-hydroxydeoxyguanosine (8-OHdG) with carotid-femoral pulse wave velocity (cf-PWV) in patients with CKD.

METHODS: Forty-three CKD patients was included in this cross-sectional study. TAC was measured from serum samples by colorimetric method and 8-OHDG was measured from urine by enzyme-linked immunoassay method. cf-PWV was measured by Doppler ultrasound as a diagnostic tool for arterial stiffness.
RESULTS: Significant correlations were found between age, estimated glomerular filtration rate (e-GFR), Hemoglobin A1C (HbA1C), serum creatinine, cystatin-C, and severity of CKD with urinary $8-\mathrm{OHdG}$ levels. Serum creatinine, cystatin- $\mathrm{C}$, severity of $\mathrm{CKD}$, age, high-density lipoprotein (HDL), e-GFR, and $\mathrm{HbA} 1 \mathrm{C}$ also had significant correlations with serum TAC levels. Other significant correlations between e-GFR, $\mathrm{HbA} 1 \mathrm{C}$, serum creatinine, cystatin-C, and severity of CKD with cf-PWV were also obtained. A significant negative correlation was obtained between serum TAC and cf-PWV with moderate levels of correlation coefficient $(\mathrm{r}=-0.504)$.

CONCLUSION: There was a negative correlation between TAC and cf-PWV, but there was no significant correlation between 8-OHdG and cf-PWV in patients with CKD.

KEYWORDS: chronic kidney disease, total antioxidant capacity, 8-hydroxydeoxyguanosine, carotid-femoral pulse wave velocity

Indones Biomed J. 2020; 12(3): 267-74

\section{Introduction}

Chronic kidney disease (CKD) is a global health problem worldwide, in which the prevalence is estimated at up to $15 \%$ in the adult population.(1) In countries with low and moderate incomes, CKD has the prevalence of $14.3 \%$ and $36.1 \%$ in the general population and high-risk populations, respectively. In these countries, people have low awareness of CKD (6-10\%).(2) The majority of patients with CKD have a higher risk of cardiovascular disease and death.
This risk increases with the progression of $\mathrm{CKD}$, marked by decreased glomerular filtration rate and increased proteinuria. $(3,4)$

In addition to traditional risk factors such as diabetes and hypertension, cardiovascular disease in CKD can also be caused by non-traditional risk factors, such as oxidative stress and chronic inflammation. This is the impact of an increase in reactive oxygen species and a decrease in antioxidant levels.(5,6) In patients with advanced CKD, increased oxidative stress is associated with increased complications, such as hypertension, atherosclerosis, 
inflammation, and anemia.(7) Some strategies performed to assess the balance of free radicals and antioxidants are the measurement of total antioxidant capacity (TAC) and 8-Hydroxydeoxyguanosine (8-OHdG). $(8,9)$

Arterial stiffness is supposed to be associated with oxidative stress through the mechanism of the release of nitric oxide synthase (NOS) and due to oxidative damage to proteins, lipids, and endothelial vascular cell DNA. (10) Inflammation can also cause an increase in arterial stiffness as inflammatory cytokines play a role in decreasing the relaxation of smooth muscle cells by reducing the bioavailability of nitric oxide (NO) and increasing endothelin-1 vasoconstrictor levels.(11) On the other hand, oxidative stress and inflammation can also cause the stiffening of the structure of blood vessels by stimulating the hyperplasia of vascular smooth muscle cells and increasing collagen synthesis.(12) Several methods have been performed to assess arterial stiffness, including pulse wave velocity (PWV).(13)

Oxidative stress is a non-traditional risk factor of CKD. The increase of oxidative stress explains the high incidence of cardiovascular disease in $\mathrm{CKD}$, in which it also becomes a new target in therapeutic interventions. Oxidative stress has been known to affect arterial stiffness, especially in patients with $\mathrm{CKD}$, and can be measured by several methods, such as malondialdehyde (MDA), superoxide dismutase (SOD), catalase, alkenals, TAC, 8-OHdG, etc. Each method has limitations and benefits, for example, MDA and alkenals have many confounding factors, i.e., sugars, amino acid, bilirubin, albumin, and hemolysis. While SOD and catalase are only able to detect enzymatic antioxidants. Total antioxidant capacity has the benefits of measuring both enzymatic and non-enzymatic antioxidant activities. The $8-\mathrm{OHdG}$ also has the benefit of measuring urinary oxidative stress and being a predictive factor for cardiovascular disease. $(14,15)$

However, until now, the correlation between TAC and 8-OHdG levels as the marker of oxidative stress with carotid-femoral PWV (cf-PWV) as the marker of arterial stiffness in patients with CKD is still unknown. Therefore, we performed this study to determine the correlation between TAC and 8-OHdG with arterial stiffness in CKD patients through the cf-PWV examination.

\section{Methods}

A cross-sectional study with 43 CKD patients at Universitas Airlangga Hospital, Surabaya, Indonesia, was conducted in
December 2019-March 2020. This study was approved by the Ethical Committee of Universitas Airlangga Hospital (Certificate No.: 189/KEH/2019).

The inclusion criteria for the participants were: patients with CKD undergoing routine treatment at Universitas Airlangga Hospital, and over 21 years old. The exclusion criteria for the participants were patients with: acute coronary syndromes (such as ST-elevation myocardial infarction (STEMI), non-STEMI (NSTEMI), and unstable angina), acute heart failure, severe infections (sepsis), cancer, and arrhythmia. The sample was collected by consecutive sampling technique.

\section{Data Collection}

Data were collected through history taking, blood pressure and anthropometry measurement, blood and urine sampling, and Doppler ultrasound. History taking was assessed by interview. Systolic and diastolic blood pressure were measured by Riester Empire N Floor Mercury Sphygmomanometer (Medaval, Dublin, Ireland). Anthropometry test was performed to measure weight, height, and abdominal circumference. Blood serum was measured for total cholesterol, low-density lipoproteins (LDL), high-density lipoproteins (HDL), creatinine, cystatin-C, Hemoglobin A1C (HbA1C), and TAC. Urine sampling was measured for 8-OHdG.

\section{Oxidative Stress Markers}

TAC was measured on the serum with the colorimetric method using Total Antioxidant Capacity Assay Kit (Cat No. PK-CA577-K274, Promokine, Heidelberg, Germany) according to the manufacturer's procedure. The absorbance of the sample was read at $570 \mathrm{~nm}$ using colorimeter Advia(C) 1800 Clinical Chemistry System (Siemens Healthineers, Erlangen, Germany) by comparing the result with the standard. The 8-OHdG was measured on urine using the enzyme-linked immunoassay method with 8-OHdG ELISA Kit (Cat. No. E-EL-0028, Elabscience, Houston, TX, USA) according to the manual instruction. The samples were measured by ELISA reader Humareader (Human Diagnostic Worldwide, Wiesbaden, Germany). The concentration of urinary $8-\mathrm{OHdG}$ was then determined by comparing the optical density (OD) of the samples to the standard curve.

\section{cf-PWV}

In the supine position, the common carotid artery was determined by B-mode as high as supraclavicular (1-2 cm from bifurcation). Simultaneous Doppler wave identification was carried out by GE Vivid 5 echocardiocraphy machine 
(GE Healthcare, Chicago, IL, USA) synchronized with electrocardiography. This examination was also carried out on the common femoral artery. To find the transit time ( $\mathrm{t}$ ), time measurements were taken from the $\mathrm{R}$ waves in the QRS complex to the Doppler wave foot using a digital caliper. Distance between the common carotid artery to the common femoral artery (d) was determined using a measuring tape. cfPWV was obtained by dividing the distance (d) by transit time difference $(\Delta \mathrm{t})$.

\section{Statistical Analysis}

Data were presented as the mean \pm SD. Spearman tests were performed to assess the correlation between variables. $p<0.05$ following a two-tailed analysis was considered to indicate statistical significance. Statistical analyses were performed using SPSS version 23 (IBM Corporation, Armonk, New York, USA).

\section{Results}

\section{Characteristics of Study Subjects}

The characteristics of the study subjects are shown in Table 1. The age range of the study subjects was 31-71 years. Male patients (58\%) outnumbered females (42\%). The most common cardiovascular risk factors found in the study subjects were hypertension (90.7\%) followed by diabetes mellitus (69.8\%) and dyslipidemia (58.1\%). The mean body mass index (BMI) of the subjects of this study was $26.51 \pm 4.63$, in which obesity $\left(\mathrm{BMI} \geq 30 \mathrm{~kg} / \mathrm{m}^{2}\right)$ is one of the cardiovascular risk factors. It was found that most of the study subjects were overweight and obese, with a BMI of more than $25 \mathrm{~kg} / \mathrm{m}^{2}$.

In the study subjects we also found other cardiovascular risk factors including increase in total cholesterol levels $(205.86 \pm 52.54 \mathrm{mg} / \mathrm{dL}), \quad$ LDL $(123.42 \pm 45.19 \mathrm{mg} / \mathrm{dL})$, creatinine $(6.91 \pm 6.79 \mathrm{mg} / \mathrm{dL}), \quad$ cystatin-C $\quad(3.80 \pm 2.63$ $\mathrm{mg} / \mathrm{L})$, and $\mathrm{HbAlc}(6.81 \pm 1.69 \%)$ and decrease in HDL $(43.88 \pm 14.79 \mathrm{mg} / \mathrm{dL})$ and e-GFR $(28.56 \pm 29.28 \mathrm{~mL} /$ $\left.\mathrm{min} / 1.73 \mathrm{~m}^{2}\right)$.

All study subjects were patients with CKD at all stages. From all study subjects, $39.5 \%$ were patients with stage 5 CKD who had undergone dialysis, while only $6.96 \%$ were patients with stage $1 \mathrm{CKD}$. The rest of the patients had stage 2-5 CKD with varying percentages. All subjects were given the same treatment in terms of basic data collection, sampling, and examination.

\section{Measurement Results of Serum TAC and urinary 8-OHdG levels}

From the present study, the mean level $\pm \mathrm{SD}$ of TAC was $1.89 \pm 0.32 \mathrm{mmol} / \mathrm{L}$ with range $1.39-2.50 \mathrm{mmol} / \mathrm{L}$, while

Table 1. Characteristics of the study subjects $(n=43)$.

\begin{tabular}{|c|c|c|}
\hline Characteristics & n (\%) & Mean \pm SD \\
\hline \multicolumn{3}{|l|}{ Male/Female } \\
\hline Male & $25(58.1)$ & \\
\hline Female & $18(41.2)$ & \\
\hline Age (years) & & $56.91 \pm 8.04$ \\
\hline History of hypertension & 39 (90.7) & \\
\hline History of diabetes mellitus & $30(69.8)$ & \\
\hline Dyslipidemia & $25(58.1)$ & \\
\hline Smoking & $11(25.6)$ & \\
\hline SBP (mmHg) & & $139.33 \pm 22.92$ \\
\hline DBP (mmHg) & & $81.05 \pm 13.21$ \\
\hline BMI $\left(\mathrm{kg} / \mathrm{m}^{2}\right)$ & & $26.51 \pm 4.63$ \\
\hline Abdominal circumference $(\mathrm{cm})$ & & $96.73 \pm 13.58$ \\
\hline Total cholesterol $(\mathrm{mg} / \mathrm{dL})$ & & $205.86 \pm 52.54$ \\
\hline $\mathrm{LDL}(\mathrm{mg} / \mathrm{dL})$ & & $123.42 \pm 45.19$ \\
\hline $\mathrm{HDL}(\mathrm{mg} / \mathrm{dL})$ & & $43.88 \pm 14.79$ \\
\hline Creatinine $(\mathrm{mg} / \mathrm{dL})$ & & $6.91 \pm 6.79$ \\
\hline e-GFR $\left(\mathrm{mL} / \mathrm{min} / 1.73 \mathrm{~m}^{2}\right)$ & & $28.56 \pm 29.28$ \\
\hline Cystatin-C (mg/L) & & $3.80 \pm 2.63$ \\
\hline $\mathrm{HbA1C}(\%)$ & & $6.81 \pm 1.69$ \\
\hline Hemoglobin $(\mathrm{g} / \mathrm{dL})$ & & $12.12 \pm 1.68$ \\
\hline
\end{tabular}

SBP: systolic blood pressure; DBP: diastolic blood pressure; BMI: body mass index; LDL: low-density lipoprotein; HDL: high-density lipoprotein; e-GFR: estimated glomerular filtration rate; HbA1C: Hemoglobin A1C. 
the mean level of urinary $8-\mathrm{OHdG}$ from the samples was $8.65 \pm 11.16 \mathrm{ng} / \mathrm{mL}$ with range $0.18-53.60 \mathrm{ng} / \mathrm{mL}$. Kolmogorov Smirnov normality test showed that the data of the two variables were not normally distributed $(p<0.05)$. Spearman correlation test was conducted to determine the correlation between the basic characteristics of the participants with the two variables. In the present study, we found a negative correlation between urinary $8-\mathrm{OHdG}$ and serum TAC $(\mathrm{r}=-0.494, p=0.001)$.

Table 2 shows the results of the correlation test. Significant positive correlations were obtained between age, e-GFR, and HbA1C with urinary 8-OHdG levels. There were also significant negative correlations between serum creatinine, cystatin- $\mathrm{C}$, and severity of CKD with urinary 8-OHdG levels. Significant positive correlations were obtained between serum creatinine, cystatin-C, and severity of CKD with serum TAC levels. In addition, significant negative correlations were also found between age, HDL, e-GFR, and HBA1C with serum TAC levels.

\section{The cf-PWV Results}

The mean $\pm \mathrm{SD}$ of the cf-PWV test in the study subjects was $16.46 \pm 8.57 \mathrm{~m} / \mathrm{s}$ with range $6.15-40.60 \mathrm{~m} / \mathrm{s}$. Kolmogorov Smirnov test on the cf-PWV variable showed that the data had an abnormal distribution $(p<0.05)$; thus, the Spearman test was performed to determine the correlation between the characteristics of the study subjects with cf-PWV.

Significant positive correlations were obtained between e-GFR and HbA1C with cf-PWV. There were also significant negative correlations between serum creatinine, cystatin-C, and severity of CKD with cf-PWV (Table 3).

\section{Correlation between TAC and 8-OHdG with cf-PWV}

Spearman correlation test was performed to determine the correlation between urinary $8-\mathrm{OHdG}$ and serum TAC with cf-PWV. A significant negative correlation was obtained between serum TAC and cf-PWV with moderate levels of correlation coefficient ( $p=0.001, \mathrm{r}=-0.504)$, whereas there was no significant association between $8-\mathrm{OHdG}$ with cfPWV ( $p=0.073, \mathrm{r}=0.276)$. This shows that the lower the TAC level was, the higher the cf-PWV was.

Based on the scatter plot graph in Figure 1, visible data plot points form a straight-line pattern from the bottom right to the top left. This shows a linear and negative relationship between the TAC variable (X-axis) with the cf-PWV variable (Y-axis). This negative relationship means that the higher the TAC value is, the lower the cf-PWV value is.

\section{Discussion}

This study involved 43 CKD patients, which were dominated by male patients. Sex hormones play a role in the progression of CKD in which estrogen acts as renoprotective factors by inhibiting oxidative stress. Male gender is associated with worse CKD progression. (16) Other than sex hormones, the progression of CKD can also be influenced by several other risk factors, i.e., hypertension, hyperglycemia, albuminuria, dyslipidemia, body mass index, lifestyle, and kidney structure.(16-18) Most of the subjects were more than 50 years old. It is said that the prevalence of CKD increases with age.(19) The high prevalence of $\mathrm{CKD}$ in older people reflects the

Table 2. Correlations between characteristics of the study subjects with urinary 8-OHdG levels and serum TAC.

\begin{tabular}{|c|c|c|c|c|}
\hline \multirow[b]{2}{*}{ Characteristics of The Study Subjects } & \multicolumn{2}{|c|}{ 8-OHdG } & \multicolumn{2}{|c|}{ TAC } \\
\hline & $\begin{array}{c}\text { Correlation } \\
\text { Coefficient (r) }\end{array}$ & Significance (p) & $\begin{array}{c}\text { Correlation } \\
\text { Coefficient (r) }\end{array}$ & Significance (p) \\
\hline Age & 0.431 & $0.004 *$ & -0.432 & $0.004 *$ \\
\hline SBP & -0.237 & 0.126 & 0.119 & 0.448 \\
\hline DBP & -0.226 & 0.145 & 0.233 & 0.133 \\
\hline BMI & 0.134 & 0.391 & -0.057 & 0.719 \\
\hline Abdominal circumference & 0.063 & 0.688 & 0.042 & 0.788 \\
\hline Total cholesterol & 0.139 & 0.374 & -0.043 & 0.787 \\
\hline LDL & 0.085 & 0.589 & -0.094 & 0.547 \\
\hline HDL & 0.082 & 0.601 & -0.306 & $0.046^{*}$ \\
\hline Creatinine & -0.537 & $0.000 *$ & 0.801 & $0.000 *$ \\
\hline e-GFR & 0.517 & $0.000^{*}$ & -0.789 & $0.000 *$ \\
\hline Cystatin-C & -0.56 & $0.000 *$ & 0.749 & $0.000 *$ \\
\hline Severe CKD $\left(\mathrm{eGFR}<30 \mathrm{~mL} / \mathrm{min} / 1.73 \mathrm{~m}^{2}\right)$ & -0.399 & $0.008^{*}$ & 0.568 & $0.000^{*}$ \\
\hline $\mathrm{HbA1C}$ & 0.485 & $0.001 *$ & -0.336 & $0.028 *$ \\
\hline
\end{tabular}

*Significant correlation, tested with Spearman test. 
Table 3. Correlation test between characteristics of the study subjects and cf-PWV.

\begin{tabular}{lcc}
\hline \multicolumn{1}{c}{ Characteristics of The Study Subjects } & Correlation Coefficient (r) & Significance (p) \\
\hline cf-PWV & & \\
Age & 0.287 & 0.062 \\
SBP & 0.217 & 0.162 \\
DBP & 0.058 & 0.712 \\
Abdominal circumference & -0.017 & 0.915 \\
BMI & -0.007 & 0.963 \\
Total cholesterol & -0.029 & 0.856 \\
LDL & -0.044 & 0.779 \\
HDL & 0.13 & 0.406 \\
Creatinine & -0.451 & $0.002^{*}$ \\
e-GFR & 0.41 & $0.006^{*}$ \\
Cystatin-C & -0.376 & $0.013^{*}$ \\
Severe CKD $\left(\mathrm{eGFR}<30 \mathrm{~mL} / \mathrm{min} / 1.73 \mathrm{~m}^{2}\right)$ & -0.341 & $0.025^{*}$ \\
HbA1C & 0.417 & $0.005^{*}$ \\
\hline
\end{tabular}

cf-PWV: carotid-femoral pulse wave velocity; SBP: systolic blood pressure; DBP: diastolic blood pressure; BMI: body mass index; LDL: low-density lipoprotein; HDL: high-density lipoprotein; e-GFR: estimated glomerular filtration rate; CKD: chronic kidney disease; HbA1C: Hemoglobin A1C.

influence of different risk factors for $\mathrm{CKD}$, such as diabetes and hypertension in older people.(20)

Significant positive correlations were obtained between serum creatinine, cystatin-C, and severity of CKD with serum TAC levels. Prior study stated that the increase in TAC in uremic patients tends to depend on the increase in uric acid levels.(21) Meanwhile in another study, it was mentioned that uric acid in circulation is a major antioxidant and can help protect against oxidative damage by free radicals.(22) In addition, this study also found significant negative correlations between age, HDL, and HBA1C with serum TAC levels. The decrease of TAC in the elderly is associated with the decrease in antioxidant capacity and the increase in oxidative stress with age.(23) Also, the condition of dyslipidemia and chronic hyperglycemia will increase oxidative stress by stimulating the release of reactive

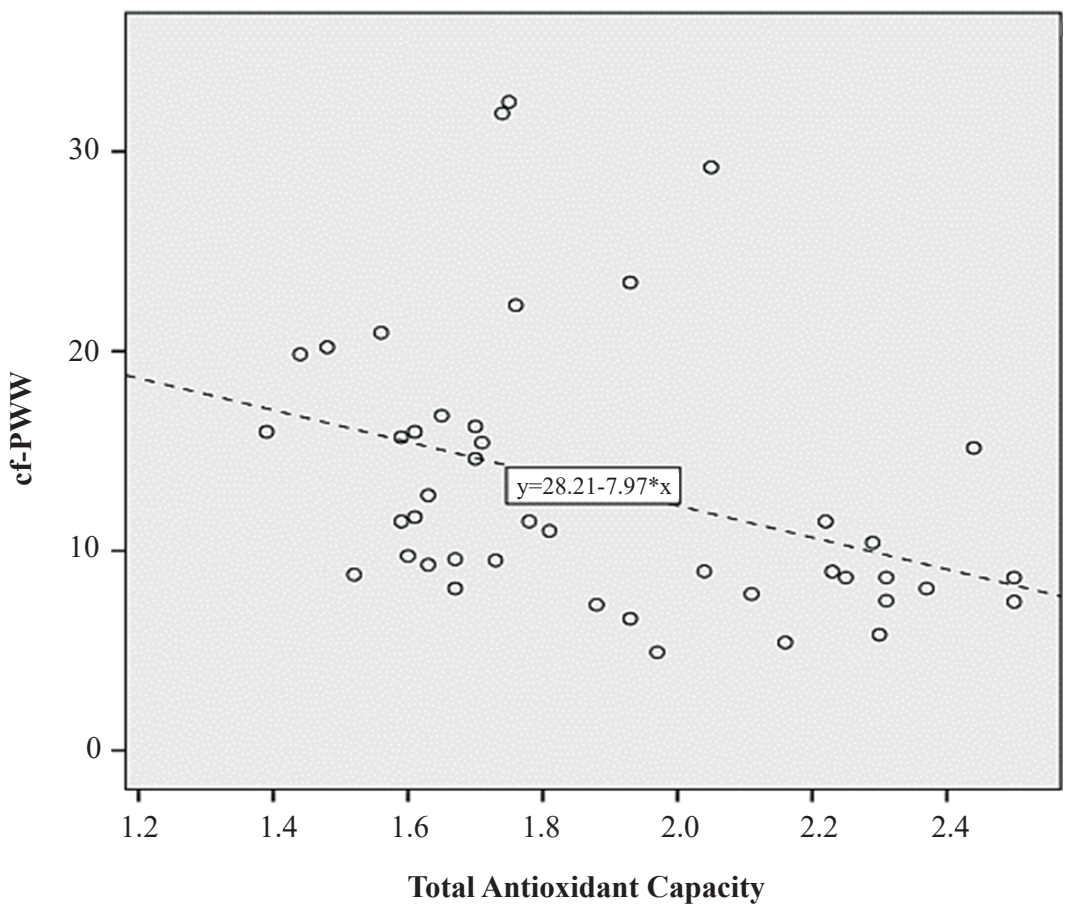

Figure 1. Scatterplot showing the correlation between TAC with cf-PWV. 
oxygen species.(24) This study found that urinary 8-OHdG was independently correlated with serum TAC. Decreased TAC levels, which reflect to increased oxidative stress, may be the reason of increased urinary 8-OHdG in CKD patients. In the level of nucleic acid, $8-\mathrm{OHdG}$ is a marker that indicates DNA damage due to oxidative stress. In this study, significant positive correlations were obtained between age, e-GFR, and $\mathrm{HbAlc}$ with urinary $8-\mathrm{OHdG}$ levels. This is consistent with previous study which states that urinary 8-OHdG levels have a significant positive correlation with HbA1C.(25) Correspondingly, the increase of $8-\mathrm{OHdG}$ and various other oxidative stress markers were also found to increase with age.(26) In this study, the level of urinary 8-OHdG was significantly higher in the lower CKD stadiums or pre-dialysis conditions. This is in line with the previous study which also found a significant increase in oxidative markers as well as a deficit of antioxidant defense in pre-dialysis populations.(27)

The mechanism underlying arterial stiffness in patients with CKD is a complex process. It involves vascular calcification, which is associated with chronic kidney disease-mineral and bone disorder (CKD-MBD). (28) CKD-MBD is a complex entity consisting of several abnormalities in mineralization, including hyperphosphatemia, hypercalcemia, hypocalcemia, and hyperparathyroidism.(29) Deposition of calcium salts on tunica intima turns to be a part of atherosclerosis, whereas deposition of calcium salts on tunica media leads to arteriosclerosis. Both conditions occur in $\mathrm{CKD}$, in which arteriosclerosis plays the most crucial role in arterial stiffness progression.(30)

In this study, a negative correlation was obtained between TAC and cf-PWV. The decrease in TAC is associated with conditions with decreased antioxidant defense mechanisms, thereby increasing oxidative stress. The decrease of TAC and arterial elasticity in 133 patients with essential hypertension, while arterial stiffness increased in elderly patients with hypertension. The decrease of antioxidant capacity contributes to vascular damage and decreased arterial elasticity in elderly patients with essential hypertension.(31) Previous study showed that strength training programs significantly affected the oxidative stress parameters, which were positively correlated with arterial stiffness. Therefore, this finding has significant implications for cardiovascular fitness after high-intensity strength training.(32)

Increased oxidative stress and decreased ability of defense mechanisms to fight oxidative stress and inflammation can contribute to changes in vascular function.(33) Increased arterial stiffness with arteriosclerosis can result in end-organ damage by increasing hemodynamic pressure in the lining of blood vessels, especially in the parts with low impedance and high flow.(30)

The measurement of urinary $8-\mathrm{OHdG}$ in the present study subjects had no significant correlation with cf-PWV. As far as the authors know, there have been no studies linking urinary $8-\mathrm{OHdG}$ with cf-PWV in patients with $\mathrm{CKD}$. There was a significant correlation between serum $8-\mathrm{OHdG}$ and arterial stiffness measured by ba-PWV in patients with stage 2-5 CKD.(34) Another study did not find any significant correlation between urinary $8-\mathrm{OHdG}$ and cf-PWV in type 2 DM patients without hypertension, but there was a significant correlation in type $2 \mathrm{DM}$ patients with hypertension.(35) The patients are influenced by several comorbidities such as diabetes mellitus, dyslipidemia, and hypertension, all of which are related to oxidative stress. $(7,36)$ CKD itself is a condition of oxidative stress independently of the underlying conditions.(37) Hemodialysis has also been shown to play a role in increasing oxidative stress.(38) In addition, several comorbidities in research subjects with varying distribution can contribute to the progression of arterial stiffness (39), so measurement of $8-\mathrm{OHdG}$ urine is not the only oxidative stress parameter that plays a role in the progression of arterial stiffness. The $8-\mathrm{OHdG}$ is a product from the interaction between guanine in DNA and free radicals. It is usually detected in the urine or serum of the patients and, the elevated levels of $8-\mathrm{OHdG}$ have been generally acknowledged as a biomarker for oxidative damage in atherosclerosis. However, its role in the arterial stiffness is still controversial and needs further research.(40)

More evidence shows that oxidative stress is considered as an important cardiovascular risk factor in patients with CKD. Cardiovascular disease is a significant etiology of morbidity and mortality in CKD patients. Biomarker examination can be used in clinical practice to monitor the oxidative imbalance of CKD patients. It is crucial to provide optimal intervention strategies to reduce oxidative stress in CKD patients.

The limitation of this study includes the high variability of the study population related to the distribution of chronic kidney disease from the early stages to end-stage renal disease. Another limitation includes the absence of the control group from the healthy population. Further research on the role of various other oxidative stress parameters on arterial stiffness as measured by cf-PWV needs to be conducted, as well as the research on the role of antioxidants in improving arterial stiffness and prospectively reducing the risk of cardiovascular disease. 


\section{Conclusion}

There was a negative correlation between TAC and cf-PWV in patients with CKD. TAC measurement can be considered as a routine examination in CKD patients as a predictor of the risk of cardiovascular disease. In this research, there was no significant correlation between 8-OHdG and cf-PWV in patients with CKD. Even though 8-OHdG is widely known as the biomarker of arteriosclerosis, its role in the arterial stiffness still needs further research.

\section{References}

1. Matsushita K, van der Velde M, Astor BC, Woodward M, Levey AS, de Jong PE, et al. Association of estimated glomerular filtration rate and albuminuria with all-cause and cardiovascular mortality in general population cohorts: a collaborative meta-analysis. Lancet. 2010; 375: 2073-81.

2. Hill NR, Fatoba ST, Oke JL, Hirst JA, O'Callaghan CA, Lasserson DS, et al. Global prevalence of chronic kidney disease - a systematic review and meta-analysis. PLoS One. 2016; 11: e0158765. doi: 10.1371/journal.pone.0158765.

3. Whitman IR, Feldman HI, Deo R. CKD and sudden cardiac death: Epidemiology, mechanisms, and therapeutic approaches. J Am Soc Nephrol. 2012; 23: 1929-39.

4. Wright J, Hutchison A. Cardiovascular disease in patients with chronic kidney disease. Vasc Health Risk Manag. 2009; 5: 713-22.

5. Cachofeiro V, Goicochea M, De Vinuesa SG, Oubĩa P, Lahera V, Lũo J. Oxidative stress and inflammation, a link between chronic kidney disease and cardiovascular disease. Kidney Int. 2008; 74: S4-S9.

6. Thaha M, Kadariswantiningsih IN, Empitu MA. Association of high blood pressure with elevated oxidative stress, inflammatory marker and albuminuria in chronic kidney disease patients. J Med. 2019; 20: $12-8$.

7. Daenen K, Andries A, Mekahli D, Van Schepdael A, Jouret F, Bammens B. Oxidative stress in chronic kidney disease. Pediatr Nephrol. 2019; 34: 975-991.

8. Fraga CG, Oteiza PI, Galleano M. In vitro measurements and interpretation of total antioxidant capacity. Biochim Biophys Acta. 2014; 1840: 931-4.

9. Sova H, Jukkola-Vuorinen A, Puistola U, Kauppila S, Karihtala P. 8-Hydroxydeoxyguanosine: A new potential independent prognostic factor in breast cancer. Br J Cancer. 2010; 102: 1018-23.

10. Silva IVG, De Figueiredo RC, Rios DRA. Effect of different classes of antihypertensive drugs on endothelial function and inflammation. Int J Mol Sci. 2019; 20: 5-9.

11. Ismaeel A, Brumberg RS, Kirk JS, Papoutsi E, Farmer PJ, Bohannon WT, et al. Oxidative stress and arterial dysfunction in peripheral artery disease. Antioxidants. 2018; 7: 1-16.

12. Zanoli L, Rastelli S, Inserra G, Castellino P. Arterial structure and function in inflammatory bowel disease. World J Gastroenterol. 2015; 21: 11304-11.

13. Ben-Shlomo Y, Spears M, Boustred C, May M, Anderson SG, Benjamin EJ, et al. Aortic pulse wave velocity improves cardiovascular event prediction: An individual participant metaanalysis of prospective observational data from 17,635 subjects. $\mathrm{J}$ Am Coll Cardiol. 2014; 63: 636-46.
14. Marrocco I, Altieri F, Peluso I. Measurement and clinical significance of biomarkers of oxidative stress in humans. Oxid Med Cell Longev. 2017; 2017: 1-32.

15. Ho E, Karimi Galougahi K, Liu CC, Bhindi R, Figtree GA. Biological markers of oxidative stress: Applications to cardiovascular research and practice. Redox Biol. 2013; 1: 483-91.

16. Valdivielso JM, Jacobs-Cachá C, Soler MJ. Sex hormones and their influence on chronic kidney disease. Curr Opin Nephrol Hypertens. 2019; 28: 1-9. doi: 10.1097/MNH.0000000000000463.

17. Duru OK, Li S, Jurkovitz C, Bakris G, Brown W, Chen SC, et al. Race and sex differences in hypertension control in CKD: results from the Kidney Early Evaluation Program (KEEP). Am J Kidney Dis. 2008; 51: 192-8.

18. Gómez-Marcos MÁ, Recio-Rodríguez JI, Gómez-Sánchez L, AgudoConde C, Rodríguez-Sanchez E, Maderuelo-Fernandez JA, et al. Gender differences in the progression of target organ damage in patients with increased insulin resistance: The LOD-DIABETES study. Cardiovasc Diabetol. 2015; 14: 132. doi: 10.1186/s12933015-0293-1.

19. Coresh J, Selvin E, Stevens LA, Manzi J, Kusek JW, Eggers P, et al. Prevalence of chronic kidney disease in the United States. J Am Med Assoc. 2007; 298: 2038-47.

20. Prakash S, O'Hare AM. Interaction of aging and chronic kidney disease. Semin Nephrol. 2009; 29: 497-503.

21. Bergesio F, Monzani G, Ciuti R, Pinzani P, Fiaschi N, Priami F, et al. Total antioxidant capacity (TAC): is it an effective method to evaluate the oxidative stress in uraemia? J Biolumin Chemilumin. 1998; 13: 315-9.

22. Fabbrini E, Serafini M, Colic Baric I, Hazen SL, Klein S. Effect of plasma uric acid on antioxidant capacity, oxidative stress, and insulin sensitivity in obese subjects. Diabetes. 2014; 63: 976-81.

23. Muralidharan N, Bhat T, N SK. A study on effect of ageing on the levels of total antioxidant and lipid peroxidation. Int J Contemp Med Res. 2017; 4: 8-10.

24. Ganjifrockwala FA, Joseph JT, George G. Decreased total antioxidant levels and increased oxidative stress in South African type 2 diabetes mellitus patients. J Endocrinol Metab Diabetes South Africa. 2017; 22: 21-5.

25. Dong QY, Cui Y, Chen L, Song J, Sun L. Urinary 8-hydroxydeoxyguanosine levels in diabetic retinopathy patients. Eur J Ophthalmol. 2008; 18: 94-8.

26. Syslová K, Böhmová A, Mikoška M, Kuzma M, Pelclová D, Kačer P. Multimarker screening of oxidative stress in aging. Oxid Med Cell Longev. 2014; 2014: 562860. doi: 10.1155/2014/562860.

27. Montesa MJP, Rico MAG, Salguero MAS, Maicas IT, Muñoz MCT, Tormo GS, et al. Study of oxidative stress in advanced kidney disease. Nefrologia. 2009; 29: 464-73.

28. Waziri B, Duarte R, Naicker S. Chronic kidney disease-mineral and bone disorder (CKD-MBD): current perspectives. Int J Nephrol Renov Dis. 2019; 12: 263-76.

29. Vikrant S, Parashar A. Prevalence and severity of disordered mineral metabolism in patients with chronic kidney disease: A study from a tertiary care hospital in India. Indian J Endocrinol Metab. 2016; 20: 460-7.

30. Lioufas N, Hawley CM, Cameron JD, Toussaint ND. Chronic kidney disease and pulse wave velocity: A narrative review. Int J Hypertens. 2019; 2019: 9189362. doi: 10.1155/2019/9189362.

31. Cao J, Wang HY. Association between total antioxidant status and atherosclerosis in elderly patients with essential hypertension. Chinese J Cardiol. 2013; 41: 857-61.

32. Jürgenson J, Serg M, Kampus P, Kals J, Zagura M, Viru M, et al. Oxidative stress parameters and its associations with arterial stiffness in competitive powerlifting athletes after 12-week 
supervised strength training. J strength Cond Res. 2019; 33: 181622.

33. Napoli C, de Nigris F, Williams-Ignarro S, Pignalosa O, Sica V, Ignarro LJ. Nitric oxide and atherosclerosis: An update. Nitric Oxide - Biology and Chemistry. 2006; 15: 265-79.

34. Dalfino G, Simone S, Porreca S, Cosola C, Balestra C, Manno C, et $a l$. Bone morphogenetic protein-2 may represent the molecular link between oxidative stress and vascular stiffness in chronic kidney disease. Atherosclerosis. 2010; 211: 418-23.

35. Kotani K, Yamada T. Association between urinary 8-OHdG and pulse wave velocity in hypertensive patients with type 2 diabetes mellitus. Singapore Med J. 2014; 55: 202-8.

36. Chade AR, Lerman A, Lerman LO. Kidney in early atherosclerosis. Hypertension. 2005; 45: 1042-9.

37. Duni A, Liakopoulos V, Roumeliotis S, Peschos D, Dounousi E.
Oxidative stress in the pathogenesis and evolution of chronic kidney disease: Untangling ariadne's thread. Int J Mol Sci. 2019; 20: 3711. doi: 10.3390/ijms20153711.

38. Ogunleye A, Akinbodewa AA, Adejumo OA, Oluwafemi TT, Akinfaderin DA. Changes in antioxidant status associated with haemodialysis in chronic kidney disease. Ghana Med J. 2018; 52: 29-33.

39. Miao R, Wu L, Ni P, Zeng Y, Chen Z. The comorbidity of increased arterial stiffness and microalbuminuria in a survey of middleaged adults in China. BMC Cardiovasc Disord. 2018; 18: 83. doi: 10.1186/s12872-018-0817-1.

40. Huh JY, Son DJ, Lee YJ, Lee JH, Kim BY, Lee HM, et al. 8-Hydroxy2-deoxyguanosine prevents plaque formation and inhibits vascular smooth muscle cell activation through Rac1 inactivation. Free Radic Biol Med. 2012; 53: 109-21. 